\section{Realidades y desafíos contemporáneos de la cooperación sur-sur en América Latina y el Caribe}

\section{Contemporary realities and challenges of the south-south cooperation in Latin America and the Caribbean}

\author{
DOI: 10.5752/P.2317-773X.2016v4.n2.p79
}

Giuseppe Lo Brutto ${ }^{1}$

Cruz Humberto González-Gutiérrez²

Recebido em: 10 de outubro de 2016

Aprovado em: 16 de janeiro de 2017

\section{RESUMEN}

El presente artículo plantea la necesidad de identificar el actual papel de la Cooperación Sur-Sur en América Latina y el Caribe, así como sus limitaciones y potencialidades. Esto debido a que en los últimos años este tipo de cooperación se ha visto caracterizada por un creciente isomorfismo conceptual que ha difuminado el espíritu solidario que marcó su auge en los primeros años de este milenio, convirtiéndole en un instrumento de posicionamiento de nuevos protagonismos y/o reposicionamiento de donantes tradicionales, llevándole a reproducir dinámicas del paradigma de la cooperación tradicional y minimizando su capacidad de construir las condiciones para un desarrollo alternativo. Circunstancias que llevan a la necesidad imperante de replantear el rol de esta forma de cooperación a nivel regional en un contexto de reconfiguración hegemónica regional y mundial.

Palabras Clave: Cooperación Sur-Sur, Desarrollo, América Latina y el Caribe, Geopolítica.

\footnotetext{
AbSTRACT

This article raises the need to identify the current role of South-South Cooperation in Latin America and the Caribbean, as well as its limitations and potentialities. This due, in recent years this type of cooperation has been characterized by a growing conceptual isomorphism, that has blurred the spirit of solidarity which marked its boom in the early years of this millennium, turning it into an instrument of positioning of new players and/or repositioning of traditional donors, that has convey to reproduce the dynamics of the traditional paradigm of cooperation and minimize its ability to build conditions for an alternative development. Circumstances that lead to an urgently need to rethink the role of this form of cooperation in a regional level, within a context of regional and global hegemonic reconfiguration.
}

Keywords: South-South Cooperation, Development, Latin America and the Caribbean, Geopolitics.
Dossier: Cooperación Sur-Sur y procesos de integración en América Latina, frente al cambio de ciclo político regional. Coordinado por Giuseppe Lo Brutto y Rafael Dominguez Martin, coordinadores del grupo de investigación en CSS e Integraciones regionales de REEDES

1. Doctor en Economía Política del Desarrollo y profesor-investigador titular del Posgrado en Sociología del Instituto de Ciencias Sociales y Humanidades "Alfonso Vélez Pliego" (ICSyH), de la Benemérita Universidad Autónoma de Puebla (BUAP). ORCID: orcid.org/00000002-8126-5013

2. PhD Student in Political Science and International Relations at University of Warsaw. ORCID: orcid.org/0000-0002$\underline{\text { 9641-6515 }}$ 
Introducción

Ante el escenario complejo que plantea esta segunda década del Siglo XXI, América Latina y el Caribe (ALyC) se yerguen frente a un panorama complicado en la esfera geoeconómica y geopolítica internacional, que ha llevado a la reconfiguración de los elementos que en años anteriores potencializaron su posibilidad de plantear dinámicas alternativas para lograr su desarrollo y emancipación de la influencia hegemónica de Occidente. Entre sus detonantes inmediatos es posible identificar: las actuales dinámicas de integración en la región, derivadas de la mayor presencia de actores externos; la caída de los precios del petróleo y otros commodities con respecto a la década anterior; la caída del crecimiento económico en aquellos países que lideraron ese nuevo auge de la Cooperación Sur-Sur (CSS) en su búsqueda de alcanzar un liderazgo hegemónico regional e internacional; el cambio de ciclo político en algunos países latinoamericanos de corte progresista; así como la llegada a la presidencia de Estados Unidos (EE.UU.) del conservador Donald Trump, promotor de un nacionalismo privatizador que pone en jaque el actual modelo de globalización imperante.

En la primera parte de este trabajo, se realiza un análisis de ALyC en el contexto de la crisis de la economía mundial capitalista, para de esta manera abordar en el segundo apartado el nuevo papel que juega la CSS en la región. Finalmente, en las conclusiones señalamos que tanto el multipolarismo como el multilateralismo marcan las pautas para este nuevo entendimiento de la CSS, apuntando igualmente a que frente a esta situación se abren nuevas posibilidades para futuros planteamientos y horizontes de la CSS en ALyC.

América Latina y el Caribe ante un escenario complejo

Hoy en día, ALyC viven una profunda crisis multidimensional, derivada, por un lado, de los efectos de la crisis de la economía mundial y de la manera en que está afecta a los países en desarrollo; y por el otro, debido al estancamiento del proceso político, económico y social de los gobiernos progresistas que ponen a la región en un nuevo momento histórico de reconfiguración e incertidumbre. Aunado a esto, la nueva política exterior del presidente Trump hacia la región, la cual podría en el corto plazo condicionar su avance hacia la consolidación de su autonomía.

En cuanto a los efectos de la crisis de la economía mundial, es importante señalar los bajos niveles de crecimiento económico de aquellos países de la región que más protagonismo tuvieron durante la primera década del siglo XXI (ver Gráfico 1).

Estos datos muestran como Brasil y Venezuela tuvieron una disminución de su crecimiento económico a partir de 2014, con un índice negativo en 2015. De la misma manera se observa como Ecuador y Bolivia tienden a bajar su índice de crecimiento durante el mismo periodo, mientras que Argentina se mantiene por debajo del 1\%. Fluctuaciones que ponen en consideración el hecho de que los países que apuntaron más hacia la construcción de un espacio regional alternativo, han enfrentado en estos últimos dos años problemas económicos que se han evidenciado paralela- 
Grafico 1 - Crescimiento del PIB Latinoamericano; Países selecionados (\% anual)

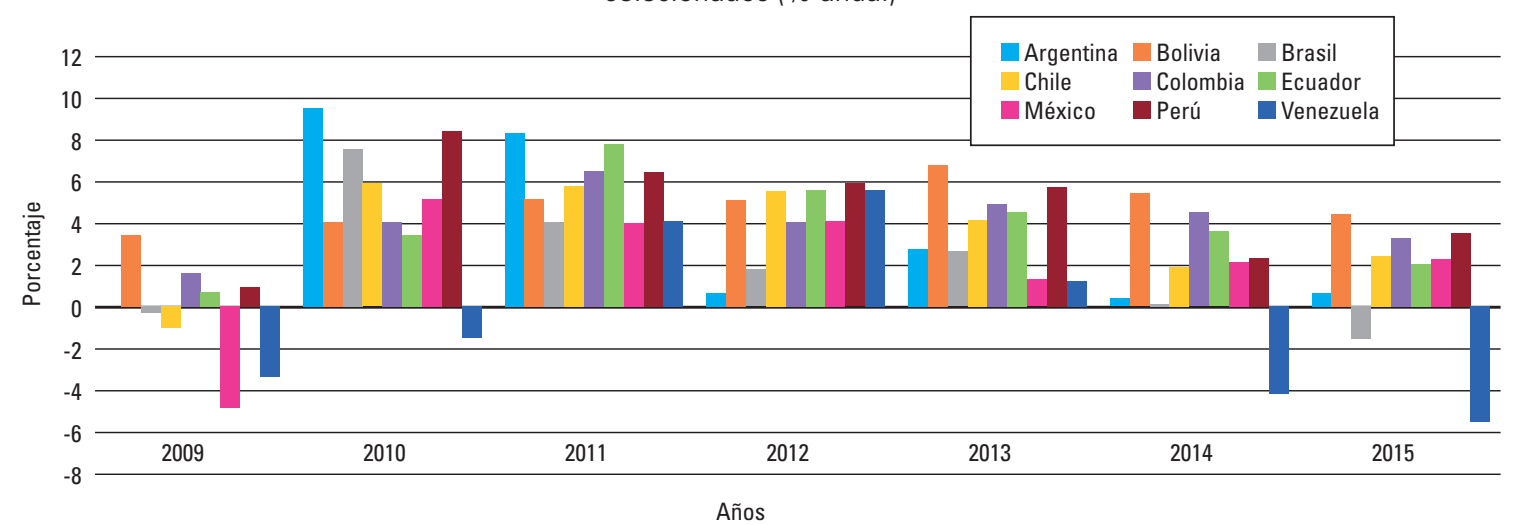

Fuente: Elaboración propia con base a datos de la CEPAL y el Banco Mundial³.

mente a sus tensiones sociales. Por otro lado, México, Chile y Perú registran avances, con la excepción de Colombia que ha visto reducido su índice de crecimiento en 2015, aunque en niveles no muy significativos. Hay que señalar que estos países (con excepción de Colombia) son parte del Acuerdo Transpacífico de Cooperación Económica (TPP por su nombre en inglés Trans Pacific Partership) que tiene en la región su expresión en la Alianza del Pacífico, la cual representa la voluntad de los Estados Unidos por adquirir una mayor fuerza en ALyC, así como en la región Asia-Pacífico. Sin embargo, con base en las recientes declaraciones del nuevo presidente electo norteamericano Donald Trump, podríamos esperar un cambio de estrategia con relación a este acuerdo, mismo que podría llevar a la reconfiguración de los objetivos planteados desde su creación.

Esta caída en el crecimiento económico regional pone de manifiesto un nuevo escenario en cuanto a la agenda del desarrollo y su respectivo impulso cada vez más marcado por las empresas transnacionales y el menor papel del Estado en la economía. Mostrando una imagen desesperanzadora en la cual pareciera que el posneoliberalismo ${ }^{4}$ quedara solamente en los escritos de aquellos académicos que hicieron de este concepto una esperanza frente a la pesadilla neoliberal, brindando a las relaciones sur-sur latinoamericanas la perspectiva de un nuevo camino por recorrer que apuntara a una renovada integración pos-hegemónica, y haciendo de ALyC un espacio regional fragmentado y a la vez compartido con el claro objetivo de darle a este continente un lugar más importante en la esfera global.

En este nuevo contexto la mayor y en parte diferente injerencia de los EE.UU. y por el otro lado, la mayor y creciente presencia de China en la región, así como el cambio político que en estos últimos meses va reconfigurando aquel espacio de izquierda y centro izquierda que la caracterizó durante la primera década del siglo XXI, estarían en conjunto delineando el futuro de las relaciones sur-sur latinoamericanas. Es por ello, que consideramos que la cooperación sur-sur va cambiando y adaptándose a los cambios políticos, económicos y sociales tanto a nivel regional como global, adquiriendo características conceptuales y una nueva retórica que reflejan el nuevo panorama internacional. Todo ello, en un contexto en el que la agenda del desarrollo o mejor dicho del desarrollo
3. Banco Mundial (2016) "Crecimiento del PIB (\% anual), datos.bancomundial.org, recuperado de: http://datos. bancomundial.org/indicador/NY.GDP. MKTP.KD.ZG

CEPAL (2016) “América Latina y el Caribe: Tasas de variación del producto interno bruto, cepal.org, recuperado de: http://www.cepal.

4. Expresa la ruptura de la hegemonía neoliberal en la región, al rechazar el posicionamiento fáctico del Consenso de Washington. 
GRAFICO 2 - Promedio Anual de Precios Internacionales del Petróleo.

Países Seleccionados (dólares)

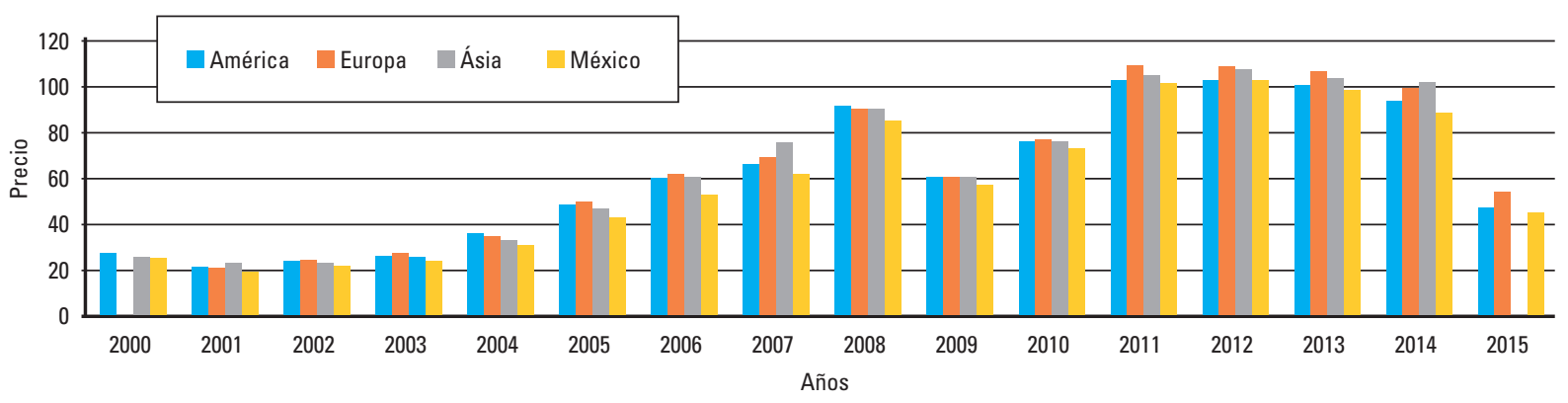

Fuente: Elaboración propia con base a datos del Centro de Estudios de las Finanzas Públicas (2006) “Precios Internacionales del petróleo 2000 2015", cefp.gob. Disponible en: www.cefp.gob.mx/intr/bancosdeinformacion/cortoplazo/.../im019.xls

5. Algunos organismos internacionales como la Organizaciones de Naciones Unidas plantean el año 2030 como el plazo para su materialización. Más detalles: http://www.un.org/sustainabledevelopment/es/la-agenda-de-desarrollo-sostenible/

6. La OPEP acordó disminuir su producción a un rango entre 32.5 millones y 33 millones de barriles de petróleo por día, (fuente Agencias Reuters y Bloomberg del 29 de septiembre de 2016). Es la primera vez en ocho años que la OPEP logra un acuerdo para recortar su producción y el convenio se haría efectivo en noviembre de 2016 en su reunión anual en Viena (Austria). sostenible queda cada vez más como una ilusión que se pretende materializar en el corto plazo 5 .

Es así que, con relación al escaso crecimiento económico en la región, es vital considerar la drástica disminución de los precios de los commodities a nivel mundial, entre los cuales destaca la caída del precio del petróleo (ver Gráfico 2). Para el caso del continente americano el promedio del precio del petróleo para el año 2015 fue de 47.01 dólares, lo cual significó una reducción del 50\% con respecto al año 2014. Situación que no se revertiría incluso una vez llegado el tercer trimestre del año 2016 en el que el precio del petróleo rondó alrededor de los 45 dólares por barril, manteniendo la perspectiva de un posible aumento para el año 2017 debido al recién acuerdo ${ }^{6}$ de la Organización de los Países Exportadores de Petróleo (OPEP) (EL ECONOMISTA, 2016).

Frente a este escenario ALyC viven un nuevo momento político, económico y social que tiene un efecto directo en la incipiente y heterogénea formación regional de este continente. Entre los años 2015 y 2016, es posible observar como el nuevo panorama político latinoamericano ha afectado la autonomía que los distintos mecanismos regionales habían adquirido, no sin dificultades y críticas. Es así que durante la III Cumbre de la Comunidad de Estados Latinoamericanos y Caribeños (CELAC) en enero de 2015, se planteó la necesidad de avanzar en medio de esta crisis multidimensional que algunos de sus miembros empezaban a manifestar, logrando el consenso sobre el objetivo de dar seguridad alimentaria y tratar de erradicar la pobreza y el hambre ante un modelo de libre mercado que en los últimos años principalmente propició procesos de privatización que incrementaron la estructura de desigualdad social en la región. Sin embargo, sería durante y después de la IV cumbre en enero de 2016, que se evidenciaría como este nuevo escenario político que se está construyendo en la región es un retorno a gobiernos neoliberales, como en el caso de la Argentina de Macri, y/o con gobiernos debilitados como en el caso de la coyuntura actual que llevara al usurpamiento del gobierno de Dilma Rousseff en Brasil a finales de agosto del 2016; hechos que sin duda le restan a este mecanismo autonomía e impacto, derivado de las pugnas que se están empezando a generar en la región sobre la manera de construir el nuevo proyecto latinoamericano. Además, la V cumbre en 
enero de 2017 se inaugura dentro de un nuevo contexto con relación a las dinamicas globales que hasta ahora venían dominado las relaciones políticas y económicas internacionales, abordando diversas temáticas entre las que destacan por su relevancia: la seguridad alimentaria, el problema de las drogas, la migración, el desarrollo, el bloqueo a Cuba y la agenda 20-30, entre otras.

En marzo de 2015, durante la Cumbre Extraordinaria de la Alternativa Bolivariana para los Pueblos de Nuestra América (ALBA), se denunciaron los planes renovados del capitalismo trasnacional globalizado y de las potencias extranjeras en sus intentos por desestabilizar a los gobiernos progresistas electos democráticamente por los pueblos latinoamericanos ${ }^{7}$. Posteriormente, durante la última cumbre del ALBA en marzo de 2016 se reafirmó esta postura y se hizo un pronunciamiento a favor de Venezuela frente a las sanciones que el gobierno de los Estados Unidos impuso a su gobierno. Lo cierto, es que frente a la grave crisis económica que se vive en Venezuela ${ }^{8}$ (INFOBAE AMÉRICA, 2016), el futuro de la Alianza Bolivariana está completamente sujeto a las vicisitudes de este país y a la manera en que Cuba vuelva con mayor fuerza al escenario regional latinoamericano, esta vez con una relación diferente con los Estados Unidos, derivada de la visita histórica que el presidente Barak Obama realizara a este país caribeño en 2016, o de lo contrario el "exitoso ocaso" de este organismo no logrará trascender esta fase de resistencia y esbozo de redes alternativas (BENZI, 2016, p. 77). Sin embargo, la nueva polítca del presidente Trump no deja esperar nada bueno para la isla caribeña en cuanto a las nuevas relaciones con los EE.UU. Pese a este escenario, la presencia cada vez mayor de China en Venezuela y en otros países ALBA puede ser considerada como un elemento importante para el futuro de este organismo regional.

Asimismo, a pesar que el gobierno venezolano afirma que PETROCARIBE avanza como una propuesta sólida de integración regional, en realidad se trata de un modelo que se dirige directamente a su agotamiento. Durante el último año, Venezuela ha renegociado la cartera de pasivos para recibir parte del pago de este financiamiento, a corto y mediano plazo por parte de los países firmantes a lo largo de diez años. Esto debido principalmente a que gran parte de la deuda de este acuerdo permanece en manos de países que tienen posibilidades limitadas para hacer frente a sus compromisos financieros con este país?.

En este mismo contexto la Unión de Naciones Sudamericanas (UNASUR), en septiembre de 2015 fue incapaz de generar el consenso necesario para poner fin a la crisis fronteriza por la que estaban pasando Colombia y Venezuela iniciada en julio de ese mismo año, siendo solucionada de forma bilateral y en ausencia del análisis de este organismo de integración ${ }^{10}$. Situación que pondría en evidencia las dificultades que enfrenta uno de los mecanismos que había sido hasta ese momento un referente y el motor del proceso de integración latinoamericano por la capacidad de negociación y estrategia de cooperación que poseía, hecho que suma negativamente en su escaso éxito para consolidarse actualmente como un modelo efectivo de integración suramericano. Además, la falta de asunción del principio de solidaridad entre los miembros también limi-
7. Venezolana de Televisión: “Declaración final de la IV Reunión extraordinaria del consejo político del ALBA-TCP" en vtv.gob.ve el 11 de agosto de 2015, dirección URL: http://www.vtv.gob.ve/ articulos /2015/08/11/ declaracion-final-de-la-iv-reunion-extraordinaria-del-consejo- politico-del-alba-tcp-7889. html

8. Llegando a alcanzar un nivel de inflación del 720 por ciento en el presente año. Infobae América "Venezuela: el aumento salarial de Nicolás Maduro alcanza para comprar un huevo por día" en http://www. infobae.com/2016/05/02/1808478-venezuela-el-aumento-salarial-nicolas-maduro-alcanza-comprar-un-huevo-dia/.

9. Malagón Álvarez, V.: "Petrocaribe una iniciativa que se agota" en acento. com el 20 de enero de 2016, dirección URL: http://acento.com.do/2016/ opinion/8316506-petrocaribe-una-iniciativa-que-se-agota/

10. Colprensa: "No hubo consenso para reunir a presidentes de UNASUR" en eluniversal.com.co el 15 de septiembre de 2015, dirección URL: http://www. eluniversal.com.co/colombia/no-hubo-consenso-para-reunir-presidentes-de-unasur-205933 
11. Venezolana de Televisión: "Culmina con éxito la XLIX Cumbre del MERCOSUR con importantes avances en materia de Derechos Humanos" en vtvgob.ve el 21 de diciembre de 2015, dirección URL: http://www.vtv.gob. ve/articulos/2015/12/21/culmina-con-exito-la-xlix-cumbre-del-mercosur-con-importantes-avances-en-materia-de-derechos-humanos-fotos-8307.html

12. El Economista: "Concluye IX Cumbre de la Alianza del Pacífico" en eleleconomista.com.mx, el 20 de junio de 2014, dirección URL: http://eleconomista. com.mx/economia-global/2014/06/20/ concluye-ix-cumbre-alianza-pacifico.

13. Banco Interamericano de Desarrollo: "Presidentes de Chile, Colombia, México, Perú y Presidente del BID analizan logros y perspectivas futuras de la Alianza del Pacífico", en http://www. iadb.org/es/noticias/comunicados-de-prensa/2016-07-01/cumbre-de-la-alianza-del-pacifico-2016,11511.html. ta el proceso de integración de la UNASUR, que en general ha invertido más energía en limar asperezas y resolver ciertos problemas internos de los miembros, que en fomentar formas de cooperación económica para consolidarse como un bloque regional sólido que le permita competir con otros países o bloques regionales, como los Estados Unidos, China o la Unión Europea (LO BRUTTO, CRIVELLI, 2015, p. 8).

Aunque muchos analistas coinciden en que la XLIX Cumbre del Mercado Común del Sur (MERCOSUR) en diciembre de 2015, culminó con importantes avances en materia de Derechos Humanos, se señala la diatriba que se generó entre Argentina y Venezuela sobre el respeto de estos últimos con relación al caso Leopoldo López generando una polémica al interior de este organismo. De igual manera, a pesar de las declaraciones que se hicieron sobre el proceso de paz en Siria; el derecho de Palestina a ser un Estado libre y soberano y también sobre la crisis migratoria que acontece actualmente en el Mediterráneo y en otras partes de Europa; Paraguay decidió no ser parte de la declaración conjunta sobre Medio Oriente. Asimismo, aunque se apoyó a Argentina en la exploración de petróleo en su plataforma continental en el territorio de las Islas Malvinas ${ }^{11}$, las citadas polémicas entre Argentina y Venezuela mostraron un organismo que refleja la nueva situación de disputa política que se está generando en la región. Aunado a ello, la reciente expulsión de Venezuela de este organismo pone en entredicho el avance con sentido alternativo que el MERCOSUR había presenciado a partir de la llegada del presidente Lula en Brasil y del presidente Chávez en Venezuela.

Por su parte, a casi cinco años de su creación en abril de 2011, la Alianza del Pacífico (AP) avanza como un mecanismo destacado en el desempeño económico de la región. Los mandatarios de Chile, Colombia, México y Perú, concluyeron su IX cumbre en junio de 2014 en Nayarit, México, con avances en movilidad de personas al firmar un acuerdo para que jóvenes puedan visitar y trabajar durante un año en los cuatro países socios, un encuentro que también culminó con el gobierno chileno insistiendo en reclamar un diálogo con el MERCOSUR ${ }^{12}$. De igual manera durante los trabajos de su XI Cumbre el pasado mes de julio en Puerto Varas, Chile entraría en vigor el protocolo comercial que elimina el 92\% de los aranceles para los productos que comercian entre si estas naciones, la interconexión de las cuatro ventanillas de comercio exterior reducirá significativamente costos y tiempos en sus intercambios; durante esta reunión también se establecería un fondo de fomento a PYMES por 100 millones de USD, estableciendo con ello bases firmes para la consolidación de este proceso de integración ${ }^{13}$.

A partir de la firma en febrero de 2016 de la Asociación Transpacífico pudo observarse como la inserción de los Estados Unidos en la región y en el área Asia-Pacífico había adquirido cada vez mayor fuerza, disputándole el control de esta zona a China y agrietando aquellos esquemas de integración regional que hasta ese momento habían dado a la región mayor autonomía -un proyecto latinoamericano con perspectiva mundial-. Sin embargo, como hemos podido observar, China no ha cedido la cuota de poder alcanzada en años anteriores tan fácilmente, y ha seguido creando las condiciones para mantener su protagonismo en el escenario interna- 
cional y consolidarse como el hegemón económico de frente al nuevo siglo, situación que fue evidente durante la Undécima Reunión de Jefes de Estado del G20 en Hangzhou, en la que se establecieron acuerdos para establecer un nuevo orden más incluyente y protagónico para los países emergentes basado en un nuevo tipo de globalización que no imponga una visión de desarrollo ${ }^{14}$ (CRUZ, 2016). Además, la reciente llegada de Trump a la presidencia norteamericana comportaría, por un lado, una mayor tensión en esa área derivado de la nueva política exterior agresiva del gobierno norteamericano, y por el otro, como señalamos también para el AP este nuevo escenario representaría un gran desafío dado que la salida de Estados Unidos del TPP implicaría el debilitamiento de un organismo que sin el apoyo indirecto de este país resultaría completamente agotado en cuando a la agenda y al proyecto de región que ha venido impulsando hasta ahora.

La cooperación sur-sur en América Latina y el Caribe en el contexto de crisis actual

Como señala Muhr (2014, p. 2) es partir de la segunda mitad del siglo XX que se comienza a hablar de la CSS como una forma de emancipación y liberación del denominado "Tercer Mundo", al tiempo que se consolidaban distintos acuerdos a nivel bilateral, multilateral y regional. De esta manera, con el paso del tiempo la CSS se posicionó como una herramienta útil tanto para los países en desarrollo como para las economías emergentes, contraria a la cooperación tradicional intentó alejarse del discurso de gobernabilidad y políticas estructurales de corte neoliberal para ensalzar los principios de solidaridad, horizontalidad, consenso y equidad.

En ese sentido, la CSS se configuró a lo largo de los primeros años de este siglo como una visión de la cooperación internacional basada en la solidaridad y representó un cambio cualitativo que incidió en la reconfiguración del sistema económico internacional y los intercambios que trascienden lo financiero, potenciando el intercambio de conocimientos y experiencias exitosas con relación a la política pública, de manera que no generara dependencia y que desarrollara las capacidades en cada país, según sus propios lineamientos y sin condicionantes (ROSERO, 2015, p. 7).

Sin embargo, hablar de CSS encierra algunas dificultades para su comprensión, lo cual ha dado como resultado que en los últimos años un análisis menos optimista respecto a sus alcances y potencialidades se desenvuelvan en ALyC, debido precisamente a su relación con la cuestión del "desarrollo" (BENZI, LO BRUTTO, 2013, p. 217). Tal como señala Bizzorero (2011:39) los procesos de cooperación e integración regional de América Latina y el Caribe (ALyC) han expresado las diferentes lógicas e historias en los cuales la CSS se ha gestado y ha ido evolucionando, logrando consolidar una nueva arquitectura política latinoamericana.

Es así que en los últimos 15 años se ha instaurado en ALyC un nuevo regionalismo multipolar que ya comienza a determinar las dinámicas y la evolución de los países latinoamericanos entorno al sistema de la Cooperación Internacional para el Desarrollo (CID), pero particularmente en relación a la CSS, donde resaltan las acciones de aquellos países dotados de
14. Consenso de Hangzhou. 
mayores capacidades materiales e inmateriales en la búsqueda de dirigir o imprimir su sello en la orientación de los procesos y dinámicas, con base a sus objetivos de política exterior y el rol que pretenden desempeñar dentro de la nueva arquitectura internacional (LO BRUTTO, GONZÁLEZ, 2014, p. 136-137). Es decir, nos encontramos frente a dos concepciones de la CSS que van asumiendo características y peculiaridades específicas, las cuales se insertan de manera paralela en una estructura que continua caracterizando la forma de establecer, instaurar y ejecutar acciones en un contexto regional y global cambiante, adaptándose cada vez más a las condiciones histórico-sociales que reflejan el sistema mundo capitalista. Es así que consideramos que la CSS sigue caracterizándose en la región por un creciente isoformismo conceptual.

Durante los últimos años la CSS ha crecido exponencialmente en la región. Con base en Ayllón (2015) los 18 países de ALyC que participan en la elaboración del informe de la CSS en Iberoamérica de la Secretaría General Iberoamericana (SEGIB) registraron en el año 2012 un total de 506 proyectos y 203 acciones de Cooperación Horizontal Sur-Sur bilateral, pasando en 2013 a 576 proyectos y 399 acciones. Entre 2010 y 2013 se realizaron 2197 proyectos con una media de 549 proyectos anuales y en el que Brasil, Argentina, México, Chile y Uruguay explican el 85\% del total de proyectos (2015, p.151). A estos datos habría que añadir lo que Venezuela y Cuba han desarrollado en los últimos 10 años que rebasa por mucho los datos anteriores. De acuerdo con Ayllón (2015), entre 1999 y 2009, Venezuela transfirió recursos a sus socios latinoamericanos con distintos grados de concesión por un valor de 36400 millones de dólares, representando este esfuerzo de ayuda en su momento un auge del 1,9\% de su PIB (2015, p.154). Además, en cuanto a la cooperación bilateral entre estos dos países hay un enorme incremento a partir del bienio 2004-2005, pasando de 346 millones de dólares a 837 millones de dólares, para alcanzar en 2007 la cifra de 1487 millones. El número de proyectos acordados -352 en 2007 en 26 áreas, 72 en 2008, 680 en 2009 y unos 100 entre 2010 y 2011 - siguiendo esta evolución. Para el año 2012 se han reportado 47 acuerdos de cooperación por un monto de 1600 millones de dólares (BENZI \& LO BRUTTO, 2014, p. 422-423). Tal como señalan los autores estas cifras no comprenden todo lo que se refiere a préstamos, inversiones e intercambios comerciales, lo que demuestra como en ALyC el dialógico político y los acuerdos comerciales tuvieron un impacto mucho más grande en términos cualitativos y cuantitativos con respecto a los otros flujos de CSS tradicional. Estos datos muestran el objetivo de mayor liderazgo regional y mundial que las políticas de cooperación e integración de Venezuela han perseguido en los últimos años en defensa del proyecto bolivariano.

En ese sentido, es posible ubicar a la CSS como producto de una heterogeneidad creciente del mundo, dada por las cambiantes condiciones económicas resultado del modelo capitalista de corte neoliberal. Esta heterogeneidad ha exigido una reestructuración geopolítica a nivel regional e internacional (LO BRUTTO, GONZÁLEZ, 2014), misma que lleva a actuar a los países bajo una "cooperación estratégica" que, como lo señala Lechini (2009), comparte principios, ideas, valores y cosmovisiones acer- 
ca de los modos de inserción internacional y el rol que deben jugar los países del sur.

En concordancia con lo anterior, la CSS nace y se reproduce en distintos contextos nacionales, resultantes de las diferencias económicas, sociales, políticas y culturales de los países que la ponen en práctica, pero que sin embargo, comparten entre ellos el sentimiento de ser el "otro" colonizado, a partir de lo cual estos actores refuerzan sus relaciones bilaterales y forman coaliciones en los foros multilaterales, llevando su participación a niveles de injerencia regional, intrarregional y multilateral global, en función de intereses políticos, económicos y comerciales. Frente a este escenario, la CSS, como herramienta de política exterior, se vale del diálogo político, la cooperación financiera y técnica para lograr acuerdos comerciales y de integración, lo que en su conjunto representa los pilares sobre los cuales se erige este tipo de cooperación que permite hacer frente a la cooperación tradicional. Cuando se menciona que estos pilares "hacen frente" a la cooperación tradicional verdaderamente se habla en su sentido literal, pues en el caso de la cooperación financiera reembolsable, el crédito concesional representa el ADN de la CSS, a partir del cual los países asiáticos y latinoamericanos oferentes de este tipo de cooperación han podido establecer el diálogo político así como acuerdos comerciales y de integración y que, pese a sus implicaciones y costos económicos para los países receptores, cambiara las reglas del juego del desarrollo internacional (DOMÍNGUEZ, 2015).

La actual fase de la CSS en ALyC parece atravesar por un momento de estancamiento, derivado básicamente por la disminuciones en los niveles de crecimiento de aquellos países que dieron a este instrumento un papel protagónico en la región a partir de la primera década del siglo XXI; por la caída de los precios del petróleo y los commodities con respecto a la década anterior; por la inserción cada vez más fuerte y a partir de ahora diferente de Estados Unidos; y por la cada vez más mayor presencia de China en la región que ha cambiado el cuadro general que teníamos apenas hace unos años en un contexto de desaceleración de la economía mundial.

Además, consideramos que estos elementos aunados a los intentos, por lo menos hasta ahora en el discurso del presidente Donald Trump, podrían cambiar el sentido de esta globalización que frente a la nueva manera de China de caracterizarla podría vislumbrar por lo menos dos escenarios: un primero, de mantenimiento del estas quo global, situación que consideramos poco probable; y, un segundo escenario, en el que se enfrentarían dos maneras de entender e imaginar esta globalización, impulsadas por las dos potencias hegemónicas actuales, China y Estados Unidos.

Esta fase de reconfiguración regional básicamente respondería a estos elementos en un contexto en el cual el diálogo político, los mega acuerdos comerciales y la desestabilización de los gobiernos progresistas que apuntan a lo que hoy se define como "fin del ciclo", bosquejan nuevos retos para los países latinoamericanos con respecto a su capacidad de insertarse con mayor o menor autonomía en las nuevas dinámicas globales de este sistema mundial capitalista. Tal como señala Llenderrozas, todo parece apuntar a que 
15. Asociación Transatlántica para el Comercio y la Inversión (TTIP por sus siglas en inglés).

16. Acuerdo en Comercio de Servicios (TISA por sus siglas en inglés).
[...] las tendencias a la construcción de bloques económicos y espacios comerciales, con el eje en Asía Pacifico, la zona más dinámica de la economía mundial, tendrá un efecto profundo en las posibilidades de inserción de los países latinoamericanos y en las alternativas disponibles. (LLENDERROZAS, 2016, p. 51).

Sin duda, la transformación de la cartografía del poder latinoamericano está relacionada con la transformación del poder a nivel mundial. Es así que, durante los últimos 15 años, China ha logrado consolidar su presencia de manera importante en ALyC, al congregar inversiones multimillonarias con múltiples acuerdos bilaterales, por tanto su presencia en la región responde a una reconfiguración de la economía mundial que establece un nuevo escenario multipolar. Asimismo, la "desaceleración de la economía China” refleja la importancia que el gigante asiático ha ido asumiendo a lo largo de estas últimas décadas y de cómo su performance económico afecta a la economía a nivel global.

Esta transformación se inscribe en un nuevo orden mundial multipolar, más regionalizado y a la vez más fragmentado, en el que los Estados Unidos han emprendido una nueva estrategia para quitarles influencia a los países emergentes en el ajedrez internacional. En este contexto destacan: las nuevas políticas comerciales que implementará el gobierno norteamericano en virtud del nuevo nacionalismo privatizador que se está impulsando, la creación del Banco Asiático de Inversión (AIIB por sus siglas en inglés), la creación de la Área de Libre Comercio de Asia Pacífico (FTAAP por sus siglas en inglés) que fue promovida por China dentro del Foro de Cooperación Económica Asia-Pacífico (APEC por sus siglas en inglés) -foro en el cual están presentes Chile, Perú y México-; y, lo que consideramos el resquebrajamiento de los esquemas comerciales globales (TTP, TTIP ${ }^{15}$ y TISA $^{16}$ ); poniendo a la región frente a un panorama de grandes retos en el que consideramos que los países de ALyC deberán apostar hacia el tipo de globalización que estas dos superpotencias están impulsando, lo que caracterizaría los esquemas de CSS que se darían a partir de esta decisión.

La declaración del presidente Trump de salir del TPP; las nuevas y difíciles relaciones que se van instaurando entre Cuba y Estados Unidos a partir de lo anterior; la nueva política exterior de los EE.UU. en ALyC; el nuevo contexto político regional derivado del debilitamiento de los "gobiernos progresistas" y de los últimos acontecimientos que ponen cada vez más a temblar la autonomía regional, apuntan por un lado a un escenario político en disputa en donde la agenda comercial neoliberal tomará cada vez mayor relevancia subordinando la visión multidimensional de los regionalismos postliberales, como por ejemplo, en el caso de las nuevas negociaciones entre Mercosur y la Unión Europea (LLENDERROZAS, 2016, p. 56); y por el otro lado, a la disputa por una nueva globalización en la que los países latinoamericanos deberán establecer una nueva manera de insertarse en las dinámicas de este capitalismo global.

El actual escenario latinoamericano nos arroja además tres elementos clave que nos permiten hacer el esfuerzo de entender hoy a la CSS en ALyC. En primer lugar, la fragmentación de los organismos de integración regional en un contexto global complejo; en segundo lugar, el panorama de la integración regional latinoamericana sigue en constante efer- 
vescencia debido también a la lucha geopolítica que tanto China, como Estados Unidos y la misma Unión Europea están desempeñando de forma distinta en la región; y finalmente, la búsqueda constante del control de los recursos naturales que en las siguientes décadas será el epicentro de lucha internacional por los commodities, al ser evidente que los distintos esquemas de integración regional no logran superar su condición neoextractivista y neodesarrollista.

Hoy el regionalismo latinoamericano añade nuevos desafíos concernientes al papel de Estados Unidos en el espacio interamericano, tales como la posibilidad de expresarse como región o integrarse bajo expresiones fragmentadas o incluso nacionales y la de conformar un camino que vislumbre una respuesta estratégica en el sistema internacional.

De esta manera, la región Asia-Pacífico representa para algunos países de ALyC una valiosa oportunidad de reposicionarse en el escenario regional, y, sobre todo, para debilitar los esquemas de integración estratégica de la primera década del siglo XXI. Más que incertidumbre, la relaciones sur-sur latinoamericanas viven un nuevo momento de convulsión en el que el factor externo está influenciando de manera directa los procesos que hasta el momento habían redibujado la cartografía de las alianzas regionales de corte progresista.

¿Hacia dónde nos dirigimos?

Frente a un escenario multipolar, interdependiente, que cuestiona la centralidad del occidente, en el que las relaciones sur-sur latinoamericanas sufren los efectos de una reconfiguración del sistema político y económico internacional, la solidaridad en la CSS queda cada vez más relegada al papel que el nuevo multipolarismo y multilateralismo le va otorgando en un sistema internacional cada vez más en mayor tensión por las pugnas que vive en su estructura.

En ese sentido, tal como señala Domínguez (2014, p.7) con un viejo sistema que se resiste a morir y el nuevo que no acaba de nacer, vivimos un momento que es a la vez de transición (BENZI, 2013) y de crisis (DOMÍNGUEZ, 2011) en el que la agenda del desarrollo no es otra cosa que la continuidad de un proyecto que ha mostrado a lo largo de estos años todo su fracaso.

Recuperando la argumentación de Nel y Taylor (2013), la solidaridad de la CSS

[...] debiera ser una actitud, basada en los sentimientos y relaciones que persiguieran el logro de fines comunes, el reconocimiento y consideración de principios morales, así como el respeto a la soberanía, la equidad y el mutuo beneficio. (NEL, TAYLOR, 2013, p. 19).

Sin embargo, en la actualidad el rompimiento con estos principios fundacionales ha llevado a categorizarle como un complemento de los mecanismos de cooperación tradicionales, ya que, al alinearse a ellos, termina por mermar su capacidad de ofrecer una vía alternativa que coadyuve al desarrollo de los países del Sur Global, reproduciendo dinámicas asimétricas en donde solo los mejores posicionados se benefician de ello. 
17. Este argumento ha sido desarrollado por Lo Brutto y González (2015), “La influencia China en la CSS latinoamericana en la segunda década de siglo XXI," consultable en http://www ciberoamericana.com/pdf/CSSLB.pdf

18. Es así que esta identidad podía considerarse como la continuidad de la crisis de identidad de la tradicional forma de cooperación Norte-Sur que se concreta con una nueva forma de relaciones Sur-Sur, poniendo al Sur global y particularmente a AL en una posición distinta; que se sustenta en la llegada a nivel nacional de gobiernos de izquierda y centro izquierda con voluntad posneoliberal con el objetivo de construir un bloque contrahegemónico que tendría su máxima expresión en tres mecanismos de integración regional: la ALBA, la UNASUR y la CELAC.
En este sentido, las dinámicas bajo las cuales se ha desarrollado la CSS llevan a cuestionar su sentido de horizontalidad y equidad en su actuar, al ser evidente el desplazamiento de su enfoque solidario por el de un interés estratégico por parte de los países del Sur, replicando los vicios del denominado Sistema Tradicional de Ayuda Occidental basado en relaciones de tipo Norte-Sur, que se someten a intereses particulares y fungen como instrumento de posicionamiento y negociación.

A lo largo de estos últimos años, tal como señala Domínguez (2014),

Toda la estrategia del CAD para atraer a los proveedores de CSS (Olivié, 2011) quedó frustrada en gran parte por la no participación en el comité directivo de la Alianza Global para la Cooperación Eficaz al Desarrollo (AGCED) de los BRICS, que han ido reforzando su coordinación global y en 2013 plantearon la creación de su propio banco de desarrollo en un claro desafío a las instituciones herederas de Bretton Woods (Cooper \& Faroq, 2013) de las que el CAD sigue siendo visto con razón como un exponente no menor [...]. (DOMÍNGUEZ, 2014, p. 15).

En ese sentido, el contraste entre aquellos países que se alinean al $\mathrm{CAD} / \mathrm{OCDE}$ y aquellos que intentan agruparse entorno a otras organizaciones que no responden a los intereses de un sistema económico y político tradicional, se hace cada día más fuerte y al mismo tiempo refleja un sistema multipolar en donde el poder parece estar repartido entre distintos polos y/o actores, en donde las relaciones entre estos polos y/o actores condicionan cada vez más las estrategias y alianzas para llevar adelante proyectos cuya hegemonía sigue siendo disputada en un ajedrez internacional más complejo.

La actual CSS en ALyC vive una cierta crisis de identidad ${ }^{17}$ con patrones claramente diferentes a los que el mismo Domínguez (2011) señalaba hace unos años. Si la identidad de la CSS en la región en la primera década del siglo XXI se caracterizó por una voluntad posneoliberal; por un intento de superar los modelos de regionalismo abierto y hacer de la recuperación de la soberanía política y económica el eje central de la construcción de un bloque contrahegemónico; y finalmente, por una resignificación de las relaciones sur-sur que llevaran a una considerable reducción de la pobreza, una mayor estabilidad económica y política, y a un intento de entender a la solidaridad de forma distinta a la ayuda tradicional ${ }^{18}$; a más una década del "giro a la izquierda", esta forma de Cooperación Sur-Sur vive igualmente una propia crisis de identidad que podría derivarse de la fragmentación de los organismos de integración regional; partiendo en primer lugar por la constante efervescencia del panorama de integración regional con la mayor presencia de China y Estados Unidos; en segundo lugar, por el intento de recuperar y volver a fortalecer la hegemonía de Estados Unidos a través del impulso de una nueva manera de establecer acuerdos comerciales y apuntar hacia una nueva globalización que estaría sustentándose en este nacionalismo privatizador que le daría a algún sector en específico un papel determinante con respecto a otros sectores del capital para impulsar una nueva manera de acumulación; en tercer lugar, por el débil pero constante rol que la Unión Europea juega en la región; y, finalmente, por la búsqueda del control de los recursos naturales con una lógica extractivista. Todo ello en un contexto de crisis multidimensional del capitalismo y el desplazamiento de la hegemonía capitalista mundial hacia el Sureste Asiático y Asia Oriental. 
En nuestra opinión, esta nueva crisis de identidad que afecta a la CSS en ALyC aleja los esperanzadores propósitos de cambio que han sido el estandarte de los diferentes mecanismos de integración regional. Ya que, tras la euforia de comienzo de milenio, hoy vemos a una CSS no solo en crisis de identidad, sino al mismo tiempo en una fase de estancamiento en un mundo cada vez más multipolar.

En conclusión, frente a estas nuevas dinámicas pensar hoy a la CSS en esta nueva globalización sur-sur implicaría revitalizar una nueva diplomacia social que diera a los movimientos el papel de decidir las relaciones supranacionales en una nueva lógica de entender la relación entre Estado, Sociedad y Mercado reconstruyendo y revitalizando las formas de solidaridad que le dieron origen.

Referencias

ALIANZA DEL PACífICO. La X Cumbre de la Alianza del Pacífico. Marzo de 2016. https:// alianzapacifico.net/la-x-cumbre-de-la-alianza-del-pacifico-en-paracas-2/ Consultado el 13 de marzo de 2016.

ÁLVAREZ, O. La CELAC: Nuevo actor regional en América Latina y el Caribe, Avales y obstáculos para lograr su consolidación. Anuario de Integración, v. 9, p.179- 206, 2012.

AYLLÓN, B. La Cooperación Sur-Sur en América Latina y Caribe. De una época dorada a una fase incierta. Anuario de Integración, v. 11, p. 134-170, 2015.

BANCO INTERAMERICANO DE DESARROLLO. Presidentes de Chile, Colombia, México, Perú y Presidente del BID analizan logros y perspectivas futuras de la Alianza del Pacífico. 1 de Julio de 2016. Disponible en <http://www.iadb.org/es/noticias/comunicados-de-prensa/2016-07-01/cumbre-de-la-alianza-del-pacifico-2016,11511.html>. Consultado el 6 Oct. 2016.

BANCO MUNDIAL. Crecimiento del PIB (\% anual). 2016. Disponible en $<$ http://datos.bancomundial.org/indicador/NY.GDP.MKTP.KD.ZG>. Consultado el 21 mar. 2016.

BENZI, D. El exitoso ocaso del ALBA. Nueva Sociedad, n. 261, 2016.

BENZI, D. Vía crucis de la cooperación internacional: ¿crisis terminal o resurrección?. Íconos, Revista de Ciencias Sociales, v. p. 9-14, 2013.

BENZI, D.; LO BRUTTO, G. Más allá de la cooperación sur-sur. Contextos, luces y sombras de la Alianza Cuba-Venezuela. En Rivera; Ayala (coordinadores) De la diversidad a la consonancia. La cooperación sur-sur latinoamericana: Estudios de México, Centro América, países donantes y ámbitos mixtos, Volumen I, BUAP \& Instituto Mora editores. 2014.

BENZI, D.; LO BRUTTO, G. La cooperación Sur-Sur en América Latina a principios del siglo XXI (un enfoque menos indulgente). En Aceves, L.; Sotomayor, H. (coordinadores) Volver al desarrollo o salir de él, Límites y potencialidades del cambio desde América Latina, BUAPEyC, México, p. 217-250, 2013

BIZZOZERO, L. América Latina a inicios de la segunda década del siglo XXI: entre el regionalismo estratégico y la regionalización fragmentada. Bras. Polít. Int., v. 54, n. 1, p. 29-43, 2011.

BRICEÑO, J. From the South American Free Trade Area to the Union of South American Nations: The Transformations of a Rising Regional Process. Latin American Policy, v. 1, n. 2, p. 208-229, 2010

CEPAL. América Latina y el Caribe: Tasas de variación del producto interno bruto. 2015. Disponible en <http://www.cepal.org/sites/default/files/pr/files/tabla-pib-estudio_economiCO_2015_0.pdf >. Consultado el 8 mar. 2016.

COLPRENSA. No hubo consenso para reunir a presidentes de UNASUR. El Universal. 15 de septiembre de 2015. Disponible en <http://www.eluniversal.com.co/colombia/no-hubo-consenso-para-reunir-presidentes-de-unasur-205933>. Consultado el 15 de sep. 2015.

COOPER, A.; FAROQU, A.B. BRICS and the Privileging of Informality in Global Governance. Global Policy, v. 4, n. p. 428-433, 2013.

CRUZ, A. A propósito del G-20 y de las críticas por su supuesta irrelevancia. Lahaine.org 28 de Septiembre de 2016. Disponible en <http://www.lahaine.org/mundo.php/apropositodelg20>. Consultado el 1 Oct. 2016. 
DOMÍNGUEZ, R. La crisis de identidad del sistema de ayuda. Fundación Carolina. 2011._Disponible en <www.fundacioncarolina.es/esES/nombrespropios/Documents/NPDomínguez1105. pdf>. Consultado el 15 feb. 2016.

DOMÍNGUEZ, R. Perspectivas de la cooperación internacional y el desarrollo sostenible después de 2015. Revista Internacional de Cooperación y Desarrollo, v. 1, n.2. 2014.

DOMÍNGUEZ, R. El tamaño importa: La financiación reembolsable en la CSS de China, India, India, Venezuela y Brasil. Cátedra de cooperación internacional y con Iberoamérica. Universidad de Cantabria. 2015.

EL ECONOMISTA. Concluye IX Cumbre de la Alianza del Pacífico. 20 de Junio de 2014. Disponible en <http://eleconomista.com.mx/economia-global/2014/06/20/concluye-ix-cumbre-alianza-pacifico>. Consultado el 20 feb. 2016.

EL ECONOMISTA. OPEP luegra acuerdo para reducir producción. 29 de Septiembre de 2016. Disponible en <http://eleconomista.com.mx/mercados-estadisticas/2016/09/29/opep-logra-acuerdo-reducir-produccion>. Consultado el 30 sep. 2016.

INFOBAE AMÉRICA. Venezuela: el aumento salarial de Nicolás Maduro alcanza para comprar un huevo por día. 2 de mayo de 2016. Disponible en $<$ http://www.infobae. com/2016/05/02/1808478-venezuela-el-aumento-salarial-nicolas-maduro-alcanza-comprar-un-huevo-dia/>. Consultado el 5 Oct. 2016.

LLENDERROZAS, E. Los desafíos de la reconfiguración: fin de ciclo y transición regional en América Latina. En Serbin, A. (coordinador) ¿Fin de ciclo y reconfiguración regional? en América Latina y las relaciones entre Cuba y los Estados Unidos. Anuario de la Integración Regional de América Latina y el Caribe (CRIES). 2016.

LECHINI, G. La cooperación sur-sur y la búsqueda de autonomía en AL ¿mito o realidad? Relaciones Internacionales, GERI-UAM, n.12, p.1-28, 2009.

LO BRUTTO, G.; GONZÁLEZ, C. El papel de la Cooperación Sur-Sur en América Latina y el Caribe como alternativa al Sistema Tradicional de Ayuda en la primera década del Siglo XXI. Centro de Estudios Latinoamericanos. Varsovia. p.119-149, 2014.

LO BRUTTO, G.; CRIVELLI, E. La Unión de Naciones Suramericanas (UNASUR) en la disputa por una integración regional en América Latina. Revista Desarrollo y Cooperación. IUDC, UCM, n.37, p. 107-115, 2015.

LO BRUTTO, G.; GONZÁLEZ, C. La influencia China en la CSS latinoamericana en la segunda década del siglo XXI. COIBA. 2015. Disponible en <http://www.ciberoamericana.com/ pdf/CSSLB.pdf>. Consultado el 21 mar. 2016.

LO BRUTTO, G.; SPATARO, A. ¿Siglo XXI, economía del terror? América Latina, Mediterráneo y Oriente Medio en un mundo en Crisis. ICSy/BUAP\& Educación y Cultura editores. México. 2016.

MALAGÓN, V. Petrocaribe una iniciativa que se agota. Acento.com. 20 de Enero de 2016. Disponible en <http://acento.com.do/2016/opinion/8316506-petrocaribe-una-iniciativa-que-se-agota/>. Consultado el 20 Ene. 2016.

MUHR, T. Beyond 'BRICS': Ten Theses on South-South cooperation in the 21st century. Beyond Global Governance: Furthering South-South Cooperation and the Role of the BRICS. Council for Social Development/Southgovnet. Nueva Delhi. 2014.

NEL, P.; TAYLOR, I. Bugger thy Neighbour? IBSA and South-South Solidarity. Third World Quarterly, v. 34, n. 6, p. 1091-1110, 2013.

OLIVIÉ, I. (coordinador). Nunca desaproveches una buena crisis. Hacia una política pública española de desarrollo. Informe Elcano: 13. 2011

VENEZOLANA DE TELEVISIÓN. Culmina con éxito la XLIX Cumbre del MERCOSUR con importantes avances en materia de Derechos Humanos. Vtvgob.ve. 21 de diciembre de 2015. Disponible <http://vtv.gob.ve/articulos/2015/12/21/culmina-con-exito-la-xlix-cumbre-del-mercosur-con-importantes-avances-en-materia-de-derechos-humanos-fotos-8307.html>. Consultado el 21 Dic. 2015.

ROSERO, G. Cooperación Sur-Sur: oportunidad para el reencuentro entre América Latina y el Caribe. Cooperamos, n. 8, p. 12-16, 2015.

VENEZOLANA DE TELEVISIÓN. Declaración final de la IV Reunión extraordinaria del consejo político del ALBA-TCP, Vtv.gob.ve. 11 de agosto de 2015. Disponible en $<$ http://www. vtv.gob.ve/articulos/2015/08/11/declaracion-final-de-la-iv-reunion-extraordinaria-del-consejopolitico-del-alba-tcp-7889.html>. Consultado el 11 ago. 2015. 